\title{
PRIMARY VAGINAL ADENOCARCINOMA WITH BICORNUATE BICOLLIS UTERUS WITH RENAL INVOLVEMENT: A RARE CASE REPORT
}

\author{
Praffulla Chandra Rai ${ }^{1}$, Sowmya Mahesh ${ }^{2}$
}

1 Post Graduate Student, Department of Radiotherapy, Assam Medical College and Hospital.

${ }^{2}$ Post Graduate Student, Department of Obstetrics \& Gynaecology, Assam Medical College and Hospital.

\begin{abstract}
\section{BACKGROUND}

Primary carcinoma of vagina is a rare entity in Gynaecological oncology. A tumour should be considered as a primary vaginal carcinoma when cervix is uninvolved. Here we are reporting a case of primary vaginal adenocarcinoma with a rare uterine malformation, right renal agenesis and enlarged left kidney. This case was confirmed by meticulous physical examination, histopathology and relevant radiological investigations which excluded the possibility of carcinoma cervix, endometrium and ovary. This patient was treated solely by chemotherapy.
\end{abstract}

\section{KEYWORDS}

Vaginal Adenocarcinoma, Bicornuate Bicollis Uterus, Vaginal Intraepithelial Neoplasia (VIN), Diethylstilbestrol (DES).

HOW TO CITE THIS ARTICLE: Rai PC, Mahesh S. Primary vaginal adenocarcinoma with bicornuate bicollis uterus with renal involvement: a rare case report. J. Evolution Med. Dent. Sci. 2016;5(25):1342-1345, DOI: 10.14260/jemds/2016/316

\section{INTRODUCTION}

Incidence of primary vaginal carcinoma is very rare (About 0.6 per 100,000 women). It constitutes about $1 \%$ of all genital malignancies.(1) Secondary vaginal carcinoma is more common than primary carcinoma and it should fulfil the criteria that the primary site of the growth should be in the vagina, cervix and vulva must not be involved and there must not be any underlying metastatic disease. Exact aetiology is unknown, but there are some predisposing factors like an underlying HPV infection, Vaginal Intraepithelial Neoplasia (VIN), cervical cancer exposure to Diethylstilbestrol (DES), radiation exposure to the genital tract, prolonged use of pessary, etc. Commonest site normally involves the upper twothird of posterior vaginal wall.(2) Squamous cell carcinoma is the commonest type; others are adenocarcinoma, melanoma and sarcoma botryoides. Treatment options are radiotherapy or surgery or chemotherapy or a combination of the three depending upon the clinical staging, location and size of the lesion.

\section{CASE HISTORY}

This patient is a 32-year-old Caucasian woman, hailing from Sivsagar, Assam, came to Gynaecology OPD in Assam Medical College and Hospital, Dibrugarh, on $5^{\text {th }}$ June 2015 with complaints of bleeding per vagina for 4-5 days. Patient had 2 living issues with last child birth 3 years back (Both issues born by normal vaginal delivery). Patient was admitted in Gynaecology ward and as per speculum examination a growth approximately $2 \mathrm{~cm}$ by $5 \mathrm{~cm}$ was seen on the anterior vaginal wall. All routine tests were done including Blood Investigations, Urine Investigations, PAP Smear and Ultrasonography (Pelvis and Whole Abdomen).

Financial or Other, Competing Interest: None.

Submission 12-02-2016, Peer Review 08-03-2016,

Acceptance 14-03-2016, Published 28-03-2016.

Corresponding Author:

Dr. Praffulla Chandra Rai,

Department of Radiotherapy,

Assam Medical College and Hospital,

Dibrugarh-786002,

Assam.

E-mail: pcr1862@gmail.com

DOI: $10.14260 /$ jemds $/ 2016 / 316$
The routine blood and urine workup was normal, but on USG patient was diagnosed to be having vaginal lesion associated with a rare uterine malformation (Bicornuate bicollis) with right renal agenesis and enlarged left kidney. On ultrasonography, no evidence of a secondary site causing metastasis was seen. The diagnosis of vaginal carcinoma was made and was Stage 3 (FIGO 2012). Its variant (Adenocarcinoma) was confirmed by Biopsy. MRI Lower Abdomen was done to see the extent of the vaginal carcinoma, which showed infiltration in surrounding periureteric fatty layer. Upon histopathological and radiological confirmation, the patient was planned for 6 cycles of chemotherapy (Paclitaxel and Cisplatin).

Each cycle was planned after a duration of 21 days. For every cycle proper consent for chemotherapy was taken and all routine blood and urine investigations were performed. After completion of 6 cycles of chemotherapy, patient was examined clinically and MRI Lower Abdomen was performed which stated that no residual disease was left. Patient was called after 3 months for followup and again a thorough clinical examination and a repeat MRI lower abdomen was done which stated that no residual disease was left. Patient has been again called after 3 months for followup.

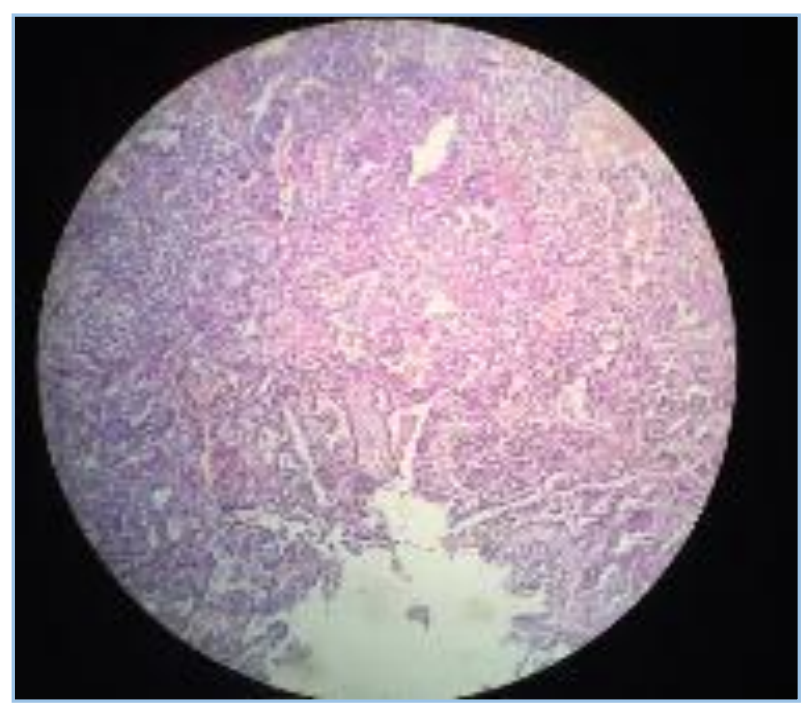

Fig. 1A 


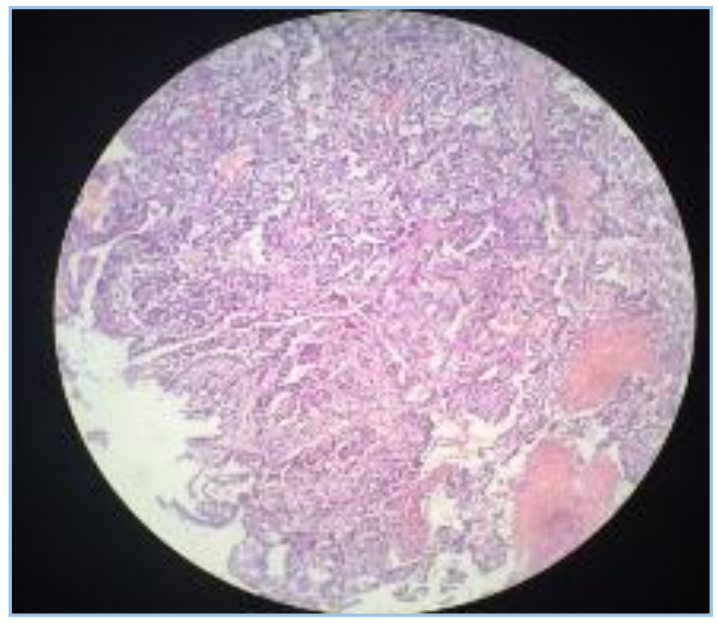

Fig. $1 B$

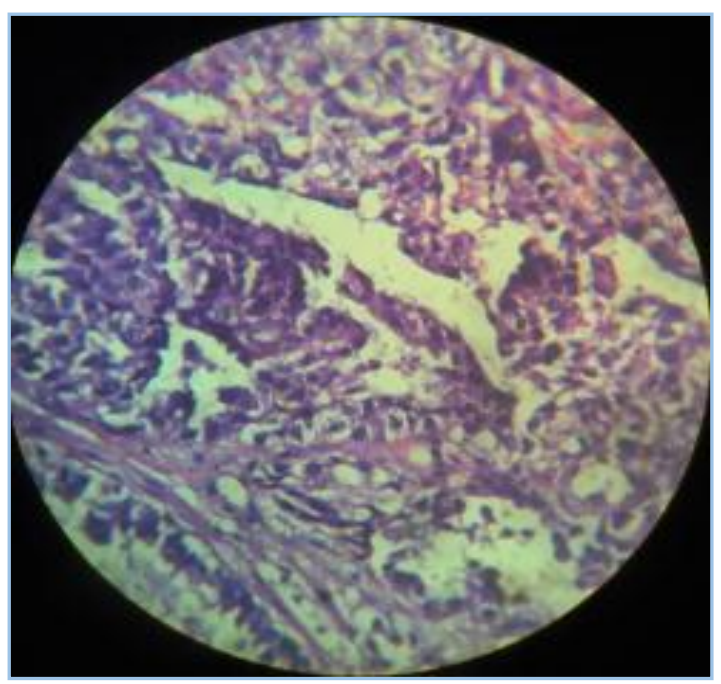

Fig. 1C

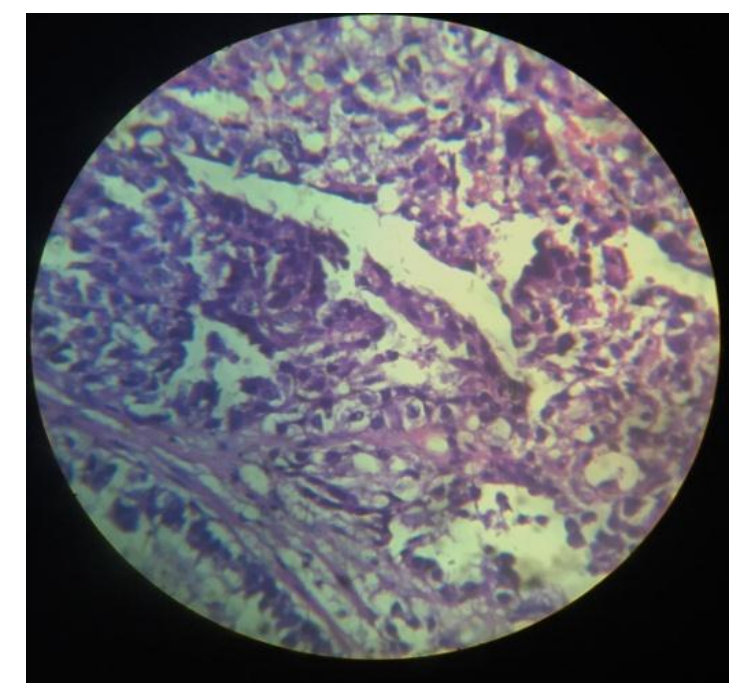

Fig. 1D

Fig. 1: $(A, B, C, D)$ Histopathology of the anterior vaginal growth biopsies demonstrating extensive adenocarcinomatous infiltrations into the stromal tissue

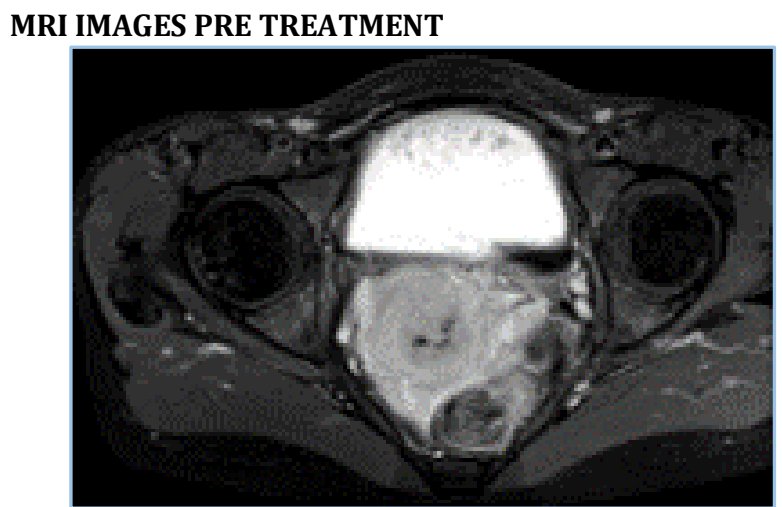

Fig. $2 A$

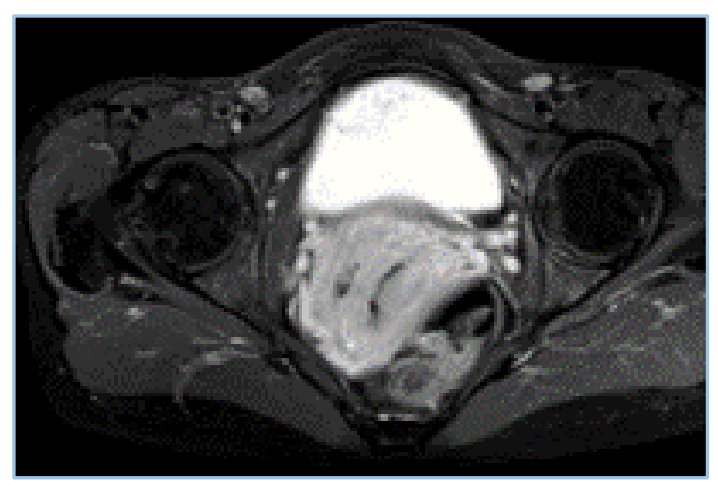

Fig. $2 B$

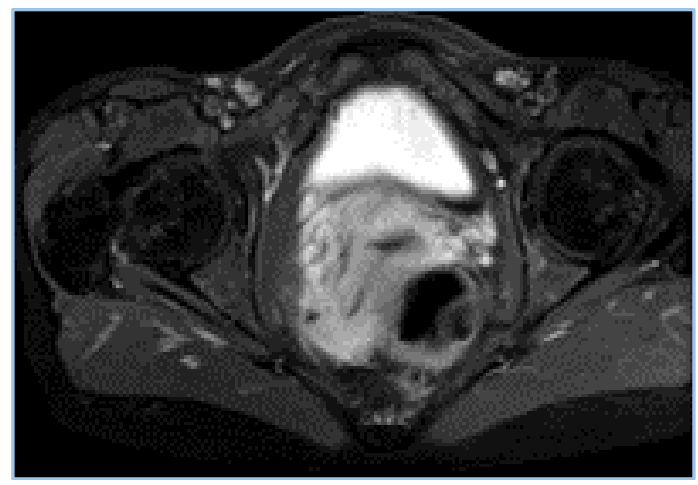

Fig. $2 C$

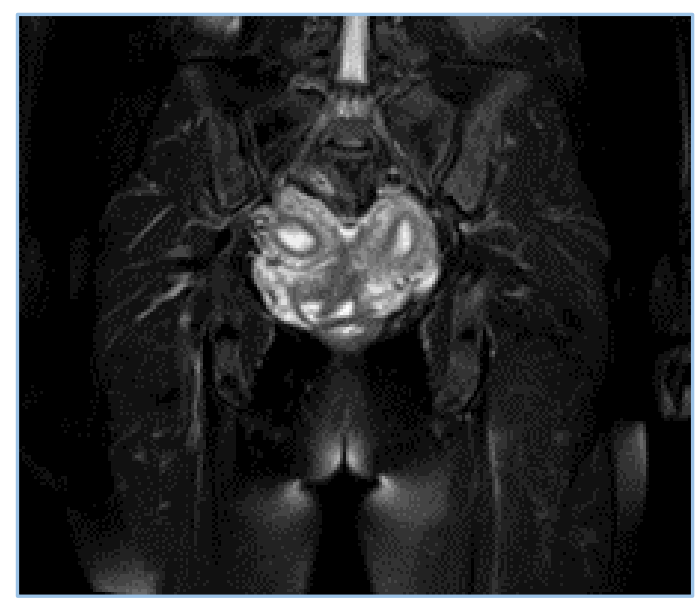

Fig. 2D

Fig. 2: (A, B \& C) Axial Post Contrast T1W MRI Images revealed a Large Irregular Heterogeneous strongly Enhancing Soft Tissue Growth in the Vaginal Region with Evidence of Regional Infiltration. (D) Coronal T2FS Image revealed Bicornuate Bicollis Uterine Anomaly 


\section{MRI IMAGES AFTER CHEMOTHERAPY}

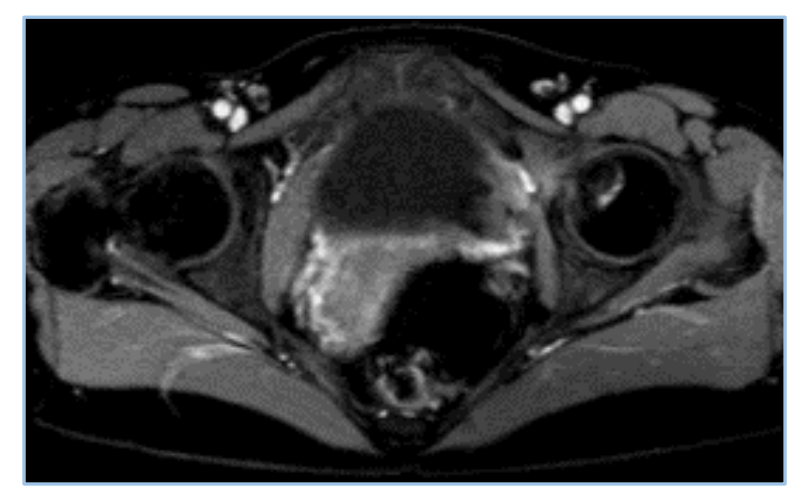

Fig. $3 A$

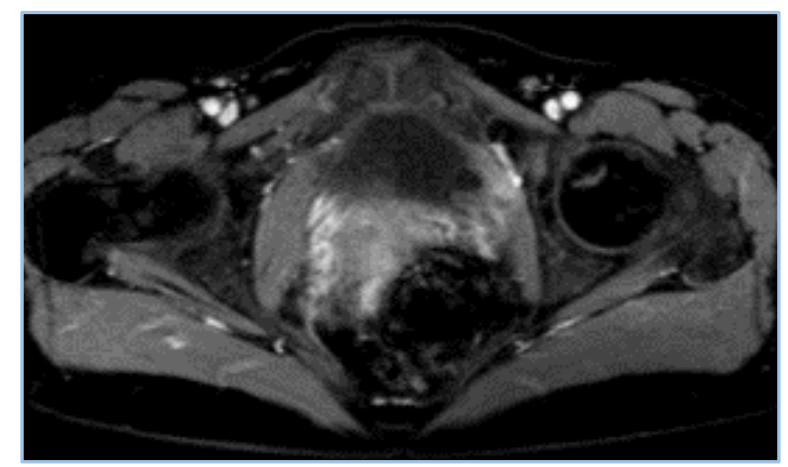

Fig. 3B

Fig. 3: (A \& B) Post Chemotherapy Axial Post Contrast T1W Images revealed Near Total Resolution of the Vaginal Growth with Minimal Non-Enhancing Residual Fibrotic Tissue

\section{MRI Images after 3 Months of Treatment}

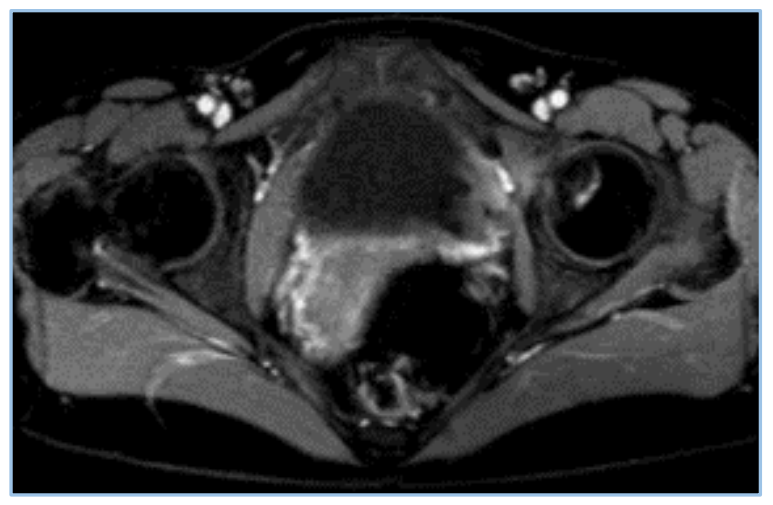

Fig. 4A

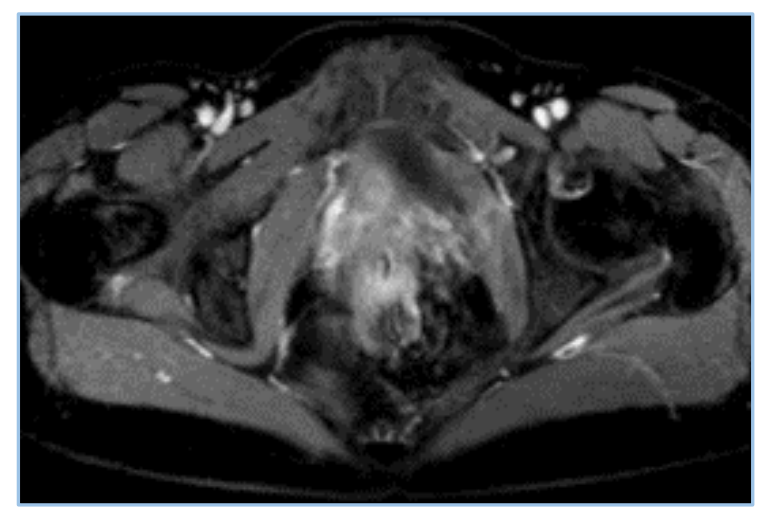

Fig. $4 B$

Fig. 4: (A \& B) Followup MRI after 3 Months. (A \& B) Axial Post Contrast T1W Images revealed no Residual/Recurrent Vaginal Growth

\section{DISCUSSION}

Carcinoma of vagina is considered as the rarest Gynaecological neoplasm. It represents less than $1-2 \%$ of Gynaecological malignancies.(1) Its incidence peaks during 60's.(2) Common factors that may increase chances of developing vaginal carcinoma are age factor (Over two-thirds of women are 60 years or older during diagnosis), an underlying smoking habit, a Human Papillomavirus (HPV) or Human Immunodeficiency Virus (HIV) infection. Other risk factors include: exposure to diethylstilbestrol (DES) as a foetus during pregnancy, vaginal adenosis, uterine prolapse, previous cervical dysplasia or a previous invasive lesion. Drinking alcohol may be contributory. Malignant diseases of vagina are usually to a primary growth elsewhere.(3) Primary vaginal carcinomas are very uncommon. Amongst the reported primary vaginal carcinoma, $92 \%$ are squamous cell carcinoma.(4)

Primary vaginal cancer should be differentiated from cancers metastatic to the vagina, which constitute the majority of cancers found in the vagina (84\%).(5) Vaginal squamous-cell carcinoma arises from the thin, flat squamous cells that line the vagina. This is the most common type of vaginal cancer with mean age of 60 years.

Malignant melanoma is the second most common cancer of the vagina accounting for $2.8 \%$ to $5 \%$ of all vaginal neoplasms.(6) Other histologic subtypes include adenocarcinoma, malignant melanomas, embryonal rhabdomyosarcoma and endodermal sinus tumour. Primary adenocarcinoma of the vagina is rare, constituting $9 \%$ of primary tumours of the vagina. The most common adenocarcinoma of the vagina is metastatic, originating from the colon, endometrium, ovary or rarely pancreas and stomach. In general, adenocarcinomas affect a younger population of women regardless of whether they were exposed to diethylstilbestrol (DES) in utero.

Adenocarcinomas may arise in wolffian rest elements, periurethral glands and foci of endometriosis.(7) Adenocarcinoma is more likely than squamous cell cancer to spread to the lungs and lymph nodes. It is found most often in women aged 30 or younger. Secondary carcinomas of the vagina are more common than primary vaginal carcinomas. Secondary or metastatic vaginal carcinomas may arise from cervical, endometrial or ovarian cancer, breast cancer, colorectal cancer or urogenital or vulvar cancer.

Often there are no symptoms and the cancer is found during a routine Gynaecologic exam. Painless vaginal bleeding and discharge are the most common symptoms of vaginal cancer. With more advanced tumours, urinary retention, bladder spasm, haematuria and frequency of urination may occur.

The incidence of lymph node metastases is directly related to the size of the tumour. Tumours from the lower third of the vagina metastasizes to inguinal lymph nodes and from the upper vagina to common iliac and presacral lymph nodes.(8)

Staging is performed by clinical examination and if indicated cystoscopy, proctoscopy and chest and skeletal radiography. Information derived from lymphangiography, CT, MRI or PET cannot be used to change the FIGO stage; however, it can be used for planning treatment. Less than $30 \%$ of vaginal cancers present at stage I.(9)

Women with stage I disease involving the upper posterior vagina may be treated by radical vaginectomy and 
pelvic lymphadenectomy. Patients with stage IV disease may be candidates for primary pelvic exenteration with pelvic and para-aortic lymphadenectomy.(10) Surgical staging with resection of enlarged lymph nodes followed by radiation may improve the control of pelvic disease. Small superficial lesions may be treated with intracavitary radiation alone.(10) Extended-field radiation may be used for vaginal cancer in a manner similar to its use for cervical carcinoma. Likewise, there is little reported experience with combination chemoradiation treatment.

\section{CONCLUSION}

A 32-year-old woman with bleeding per vagina diagnosed as a case of primary vaginal carcinoma with a uterine malformation and renal involvement. Patient was put on a chemotherapy regimen of paclitaxel and cisplatin; 6 cycles were given at an interval of 21 days.

Clinical examination and followup MRI scans revealed regression of tumour. Patient has been planned for further followup.

\section{REFERENCES}

1. Begum N, Ara I, Islam F, et al. Primary vaginal carcinoma in prolapsed uterus. J Bangladesh Coll Phys 2012;30(3):181182.
2. Batista T, Morais J, Reis T, et al. A rare case of invasive vaginal carcinoma associated with vaginal prolapse. Arch Gynecol Obstet 2009;280(5):845-848.

3. Donato A. Vaginal carcinoma. In: Santos CER, Mello ELR eds. Surgical oncology handbook. Tecmed Sao Paulo 2003;555-560.

4. Creasman WT. Vaginal cancers. Current Opinion in Obstetrics and Gynecology 2005;17(1):71-76.

5. Fu YS. Intraepithelial, invasive and metastatic neoplasms of the vagina. In: Pathology of the uterine cervix vagina and vulva. Philadelphia: Saunders, 2002;2nd ed:p 531.

6. Ballon SC, Lagasse LD, Chang $\mathrm{NH}$, et al. Primary adenocarcinoma of the vagina. Surg Gynecol Obstet 1979;149:233-237.

7. Reid GC, Schmidt RW, Roberts JA, et al. Primary melanoma of the vagina: a clinicopathologic analysis. Obstet Gynecol 1989;74(2):190-199.

8. Resella S, Muria EF, Rebeiro JU. Carcinoma of vagina associated with genital prolapsed in nullipara patient: a case report. J Bars Gynaecol 1994;104(3):63-65.

9. FIGO, International Federation of Gynecology and Obstetrics. From FIGO Annual Report. Int J Gynecol Obstet 2006;95:S29. and Int J Gynecol Obstet 2009;105:3-4.

10. Cramer DW, Cutler SJ. Incidence and histopathology of malignancies of the female genital organs in the united states. Am J Obstet Gynecol 1974;118(4):443-460. 